\title{
CONVOLUTION EQUATIONS AND HARMONIC ANALYSIS IN SPACES OF ENTIRE FUNCTIONS
}

\author{
BY
}

\author{
D. G. DICKSON
}

ABSTRACT. If $H$ is the topological space of functions analytic in the simply connected open set $\Omega$ of the plane with the topology of compact convergence, its dual may be identified with the space $E$ of functions of exponential type whose Borel transforms have their singularities in $\Omega$. For $f$ in $H$ and $\phi$ in $E,(f * \phi)(z) \equiv\left\langle f, \phi_{z}\right\rangle$ where $\phi_{z}$ is the $z$-translate of $\phi$. If $f \equiv 0$ in any component of $\Omega, f * \phi=0$ if and only if $\phi$ is a finite linear combination of monomial-exponentials $z^{p} \exp (\omega z)$ where $\omega$ is a zero of $f$ in $\Omega$ of order at least $p+1$. For such $f$ and $\psi$ in $E, f * \phi=\psi$ is solved explicitly for $\phi$. If $E$ is assigned its strong dual topology and $r(\phi)$ is the closed linear span in $E$ of the translates of $\phi$, then $r(\phi)$ is a finite direct sum of closed subspaces spanned by monomial-exponentials. Each closed translation invariant subspace of $E$ is the kernel of a convolution mapping $\phi \rightarrow f * \phi$; there is a one-to-one correspondence between such subspaces and the closed ideals of $H$ with the correspondence that of annihilators.

1. Introduction. Let $\Omega$ be a nonempty open set in the complex plane with a connected complement in the extended plane. Let $E$ be the linear space of entire functions of exponential type whose Borel transforms can be continued to $\tilde{\boldsymbol{\Omega}}$, the complement of $\boldsymbol{\Omega}$. For $f$ analytic in $\boldsymbol{\Omega}$ and $\phi$ in $E$, the convolution of $f$ and $\phi$ is defined by

$$
(f * \phi)(\zeta)=\frac{1}{2 \pi i} \int_{\gamma} f(z) \tilde{\phi}_{\zeta}(z) d z
$$

where $\tilde{\phi}_{\zeta}$ is a continuation of the Borel transform of the $\zeta$ translate $\phi_{\zeta}$ of $\phi$, and where $\gamma$ is a finite chain of simple closed curves in $\Omega$ about the singularities of the Borel transform of $\phi$. We find here the general solutions in $E$ of the equations

$$
\begin{aligned}
& f * \phi=0, \quad \text { and } \\
& f * \phi=\psi
\end{aligned}
$$

where $\psi$ is in $E$.

Received by the editors December S, 1972.

AMS (MOS) subject classifications (1970). Primary 30A20, 30A98; Secondary 30A64, 39A 15, 44A35, 46E 10 .

Key words and phrases. Convolution, entire functions of exponential type, harmonic analy sis.

Copyright 0 1974, American Mathematical Society 
When $f$ is not the zero function in any component of $\Omega$, then the solutions of (1) are the finite sums of the form

$$
\sum_{k=1}^{n} p_{k}(z) \exp \left(\zeta_{k} z\right)
$$

where each $\zeta_{k}$ is a zero of $f$ in $\Omega$ and each $p_{k}$ is a polynomial of degree less than the order of $\zeta_{k}$. For such an $f$, convolution by $f$ maps $E$ onto itself and all solutions of (2) may be written explicitly. Solutions for general $f$ are given when they exist. The subspace of $E$ spanned by the translates of (3) is the space spanned by the monomial-exponentials $z^{p} \exp \left(\zeta_{k} z\right)$ where $p$ varies from 0 to the degree of $p_{k}$.

If $H$ is the topological linear space of functions analytic in $\Omega$ with the topology of uniform convergence on compacta, then $E$ is its topological dual. $H$ is reflexive, so $E$ is assigned its strong dual topology. Our convolution is then defined in terms of the duality by $(f * \phi)(\zeta)=\left\langle f_{0} \phi_{\zeta}\right\rangle$. Let $r(\phi)$ be the closed linear span in $E$ of the translates of $\phi$. When $\Omega$ is connected, $\tau(\phi)$ is either the finite dimensional space described above or is all of $E$. In all cases, $\tau(\phi)$ is a finite direct sum of closed subspaces spanned by monomial-exponentials. $r(\phi) \neq E$ if and only if $\phi$ satisfies (1) for some $f \neq 0$. Each closed translation invariant subspace of $E$ is the kernel of some convolution mapping, and there is a one-to-one correspondence between such subspaces and the closed ideals of $H$.

The mapping from $H$ into $E$ defined by convolution with a fixed function in $E$ is examined briefly in the last section.

With a different representation of the dual of $H$, Köthe ( $[5$, p. 47] and [6, p. 434]) studied equations which are equivalent to (1) and (2) using duality. The mapping equivalent to our convolution was studied as the adjoint of the multiplication mapping $g \rightarrow g f$ of $H$ into itself. We show the equivalence by showing that multiplication is adjoint to convolution. Köthe finds solutions of the equation equivalent to (1) and shows the existence of solutions of the equation equivalent to (2). Our methods of solution are constructive and do not use a topological structure on $E$ or the fact that $H$ and $E$ are dual.

If $\Omega$ is a disk with center at the origin and radius $r$, then $E$ is the set of entire functions of exponential type less than $r$, and convolution of $\phi$ by $f(z)=$ $\Sigma a_{n} z^{n}$ has the form of the infinite order differential operator $\Sigma_{a_{n}} \phi^{(n)}(z)$. In this case, results similar to those obtained here regarding the solutions of (1) and (2) were originally given by Muggli [7] with an alternative derivation given in [3]. If $\Omega$ contains the finite set of points $\left\{\omega_{i}\right\}$ and $f(z)=\Sigma a_{i j} z^{j} \exp \left(\omega_{i} z\right)$ is a finite sum, then convolution of $\phi$ by $f$ has the form of the differencedifferential operator $\Sigma a_{i j} \phi^{(j)}\left(z+\omega_{i}\right)$. In the general case considered in this paper, $\Omega$ is not assumed to be convex or connected. 
In a sense, the problems studied here are dual to the usual studies of analytic mean-periodicity where solutions $f$ of $\langle f(w+z), \phi(w)\rangle=0$ for $\phi$ of exponential type are characterized when $\Omega$ is the entire plane making $H$ translation invariant [4], [9], or when $H$ is specialized so that the equation has meaning for $f$ in $H$ and $z$ in some restricted set [2]. In our case, $H$ is not translation invariant while $E$ is invariant.

2. Preliminaries. An open and connected set in the plane will be called a region while the complement in the plane of a set $S$ will be denoted by $\widetilde{S}$.

Generally, the topological dual $H^{\prime}$ of $H$ is identified with the set of functions that are locally analytic on the complement of $\Omega$ in the extended plane and vanish at infinity. That is, $H^{\prime}$ is the set of equivalence classes $\phi^{*}$ of function elements $(\tilde{\phi}, S)$ where $S$ is a region containing $\tilde{\Omega}, \tilde{S}$ is bounded, and where $\tilde{\phi}$ is analytic in $S$ with $\tilde{\phi}(z) \rightarrow 0$ as $z \rightarrow \infty$; two function elements are equivalent if their functions are equal in some region containing $\tilde{\Omega}$. The duality is given by $\left\langle f, \phi^{*}\right\rangle=(2 \pi i)^{-1} \int_{\gamma} f(w) \tilde{\phi}(w) d w$ where $f \in H,(\tilde{\phi}, \mathrm{S}) \in \phi^{*}$, and $\gamma$ is a finite chain of simple closed, positively oriented, rectifiable curves in $\Omega \cap S$ with $\tilde{\Omega}$ in the exterior of $\gamma$ and $\tilde{S}$ in the interior of $\gamma$; distinct curves of the chain are in distinct components of $\Omega$. These facts are detailed by Köthe in [5, p. 375].

Since the complement of $\Omega$ in the extended plane is connected, the Fourier transformation $\phi(z)=\left\langle\epsilon_{z}, \phi^{*}\right\rangle$ where $\epsilon_{z}(w)=e^{z w}$ permits an identification of $H^{\prime}$ with the set of entire functions we have denoted by $E$. Specifically, if each function of a function element in $\phi^{*}$ has the series development $\Sigma_{0}^{\infty} n ! a_{n} / z^{n+1}$ about infinity, then $\phi(z)=\Sigma_{0}^{\infty} a_{n} z^{n}$; the former series is called the Borel trans form [1] of $\phi$ and is the analytic continuation of the real Laplace transform of $\phi$. The equivalence class corresponding to a $\phi$ in $E$ is then $\phi^{*}$. With $E$ as the dual of $H$, the duality is given by $\langle f, \phi\rangle=\left\langle f, \phi^{*}\right\rangle$.

If $\left\{\Omega_{i}\right\}$ is the set of components of $\Omega$ and if $H_{i}$ is the topological linear space of functions analytic in $\Omega_{i}$ with the topology of uniform convergence on compacta, then $H=\Pi H_{i}$, the topological product of the $H_{i}$. If $E_{i}$ is the dual of $H_{i}$, then $E=\bigoplus E_{i}$, the direct sum of the $E_{i} . H$ and the $H_{i}$ are Fréchet and Montel spaces. Since they are Montel spaces, they are reflexive and we will consequently assign the strong dual topologies to $E$ and the $E_{i}$ when topological considerations are required. $E$ is then the topological direct sum of the $E_{i}$ •

If $f$ is in $H$ and $f=\left\{f_{i}\right\}$, then, as usual, $f \neq 0$ means that $f_{i} \neq 0$ for some $i$. We will write $f \# 0$ to denote the fact that $f_{i} \neq 0$ for each $i$. While theorems requiring $f \# 0$ generally follow from the case when $f \neq 0$ and $\Omega$ is connected, the proofs are the same, so they are given in the more general form.

For $\phi$ in $E$ we will say that $(\tilde{\phi}, S, \gamma)$ is $\phi$-admissible (for the duality) when $\left(\widetilde{\phi}_{0} s\right) \in \phi^{*}$ and $\gamma$ is chosen as above. $C$ will denote the set of complex 
numbers, and for $\phi$ in $E$ and $\zeta$ in $C$ the $\zeta$-translate of $\phi$ is $\phi_{\zeta}(z) \equiv \phi(z+\zeta)$. For $\psi$ and $\phi$ entire functions, the resultant or "finite" convolution of $\psi$ and $\phi$ is given by $(\psi \circ \phi)(z)=\psi(z) \circ \phi(z)=\int_{0}^{z} \psi(z-w) \phi(w) d w$.

The first two lemmas will permit the formulation of the definition of convolution and the definition of some useful functions in $H$.

Lemma 1. For $\zeta$ in $C$, the mapping $\phi \rightarrow \phi_{\zeta}$ maps $E$ onto $E$. Further, if $\phi \in E$ with $(\tilde{\phi}, S)$ in $\phi^{*}$ and $\tilde{\phi}_{\zeta}(z) \equiv e^{\zeta} \tilde{\phi}_{\phi}(z)-e^{\zeta z} \circ \phi(\zeta)$, then $\left(\tilde{\phi}_{\zeta}, S\right) \in \phi_{\zeta}^{*}$.

Proof. $\phi_{\zeta}$ is clearly of exponential type. Since the Borel transform is given by the Laplace transform for $z$ large and real, the Borel transform of $\phi_{\zeta}$ for such $z$ is given by

$$
\begin{aligned}
\int_{0}^{\infty} e^{-z t} \phi(t+\zeta) d t & =\lim _{R \rightarrow \infty} \int_{\zeta}^{\zeta+R} e^{z(\zeta-w)} \phi(w) d w \\
& =\lim _{R \rightarrow \infty} \int_{0}^{\zeta+R} e^{z(\zeta-w)} \phi(w) d w-e^{\zeta z} \circ \phi(\zeta) .
\end{aligned}
$$

The integral from 0 to $\zeta+R$ may be replaced by the sum of integrals from 0 to $R$ and from $R$ to $\zeta+R$. For $z$ greater than the type of $\phi$, the limit of the first integral is $e^{\zeta z} \widetilde{\phi}(z)$ while the limit of the second is zero. Hence $e^{\zeta z} \tilde{\phi}(z)-e^{\zeta z} \circ \phi(\zeta)$ is the Borel transform of $\phi_{\zeta}$ for $z$ of large modulus. This function $\tilde{\phi}_{\zeta}$ is analytic in $S$ since the second term is entire. Hence $\left(\tilde{\phi}_{\zeta}, S\right) \epsilon$ $\phi_{\zeta}^{*}$ and the mapping $\phi \rightarrow \phi_{\zeta}$ is into $E$. The mapping is onto $E$ since $\phi$ is the image of $\phi_{-\zeta}$.

Definition 1. For $f$ in $H$ and $\phi$ in $E$, the convolution of $f$ and $\phi$ is the function on $C$ defined by $(f * \phi)(\zeta)=\left\langle f, \phi_{\zeta}\right\rangle$.

Since the finite convolution $e^{\zeta z} \circ \phi(\zeta)$ is entire, by choosing $(\widetilde{\phi}, s, \gamma)$ to be $\phi$-admissible, it follows from Lemma 1 that

$$
(f * \phi)(\zeta)=\frac{1}{2 \pi i} \int_{\gamma} e^{\zeta w} f(w) \tilde{\phi}(w) d w=\left\langle e^{\zeta z} f(z), \phi(z)\right\rangle .
$$

If $\zeta \in \Omega$, then $e^{\zeta z} \in E$ and $\left((z-\zeta)^{-1}, C-\{\zeta\}\right)$ is in $\left(e^{\zeta z}\right)^{*}$; then $\left\langle f(z), e^{\zeta z}\right\rangle=f(\zeta)$ by Cauchy's theorem. Of course we also know that $\left\langle e^{\zeta z}, \phi(z)\right\rangle$ $=\phi(\zeta)$ for all $\zeta$ in $C$. If $t \in \Omega$ and $f \in H$, then $\left(f(z) * e^{t z}\right)(\zeta)=e^{\zeta_{t}} f(t)$ by (4).

Lemma 2. If $\psi$ and $\phi$ are in $E$ with $(\tilde{\psi}, T)$ and $(\tilde{\phi}, s)$ in $\psi^{*}$ and $\phi^{*}$ respectively, then $\psi \circ \phi$ is in $E$ with $(\tilde{\psi} \tilde{\phi}, U)$ in $(\psi \circ \phi) *$ where $U$ is the unbounded component of $T \cap S$.

Proof. The usual proof for Laplace transforms shows that the Borel 
transform of $\psi \circ \phi$ for $z$ of large modulus is $\tilde{\psi} \tilde{\phi}$, and this product is analytic in $U$ which contains $\tilde{\Omega}$.

It follows from Lemma 2 that if $\zeta \in \Omega$ and $(\tilde{\phi}, S) \in \phi^{*}$, then $(\tilde{\phi}(z) /(z-\zeta)$, $S-\{\zeta\})$ is in $\left(e^{\zeta z} \circ \phi(z)\right) *$. In particular, $e^{\zeta z} \circ \phi(z) \in E$ when $\zeta \in \Omega$ and $\phi \in E$. This observation permits the formulation of the following definition.

Definition 2. For $f$ in $H$ and $\phi$ in $E$, the function $T_{f} \phi$ from $\Omega$ into $C$ is defined by $(T, \phi)(\zeta)=\left\langle f(z), e^{\zeta z} \circ \phi(z)\right\rangle$.

Then $T, \phi \in H$. For suppose $\zeta \in \Omega$ and $(\tilde{\phi}, S) \in \phi^{*}$. Choosing $\gamma$ so that $(\tilde{\phi}, S, \gamma)$ is $\phi$-admissible and so that $\zeta$ is in the interior of $\gamma$, we have

$$
(T, \phi)(\zeta)=\frac{1}{2 \pi i} \int_{\gamma} \frac{f(w) \tilde{\phi}(w)}{w-\zeta} d w
$$

which is analytic in a neighborhood of $\zeta$.

3. Representations of solutions. In this section we will find all solutions of (1) and (2).

Theorem 1. For $f$ in $H$ the mapping $\phi \rightarrow f * \phi$ maps $E$ into E. Further, if $\phi \in E$ with $(\tilde{\phi}, S)$ in $\phi^{*}$, there exists an $\omega$ with $(\omega, S)$ in $(f * \phi)^{*}$ and $\omega=\tilde{\phi}-T, \phi$ on $\Omega \cap S$.

Proof, Suppose $(\tilde{\phi}, S) \in \phi^{*}$. For each $z$ in $S$ choose $\gamma_{z}$ so that $\left(\tilde{\phi}, S, \gamma_{z}\right)$ is $\phi$-admissible and $z$ is exterior to $\gamma_{z}$. Define

$$
\omega(z)=\frac{1}{2 \pi i} \int_{\gamma_{z}} \frac{f(w) \tilde{\phi}(w)}{z-w} d w .
$$

$\omega$ is well defined by Cauchy's theorem and is analytic in $S$. It is clear from (4) that $f * \phi$ is entire and of exponential type. For $z$ large, real, and outside of $y=\gamma_{z}$, the Borel transform of $f * \phi$ is given by

$$
\begin{aligned}
\int_{0}^{\infty} e^{-z t} \frac{1}{2 \pi i} \int_{\gamma} e^{t w} f(w) \tilde{\phi}(w) d w d t & =\frac{1}{2 \pi i} \int_{\gamma} f(w) \tilde{\phi}(w) \int_{0}^{\infty} e^{-t(z-w)} d t d w \\
& =\frac{1}{2 \pi i} \int_{\gamma} \frac{f(w) \tilde{\phi}(w)}{z-w} d w=\omega(z) .
\end{aligned}
$$

The absolute convergence of the second integral with respect to $t$ justifies the change in order of integration. Since $\omega$ is analytic in $S, f * \phi$ is in $E$.

Suppose now that $\zeta \in \Omega \cap S$. Choose $\gamma$ and $\gamma^{\prime}$ so that $\zeta$ is in the exterior of $\gamma$ and in the interior of $\gamma^{\prime}$ and so that each of $(\tilde{\phi}, S, \gamma)$ and $\left(\tilde{\phi}, S, \gamma^{\prime}\right)$ is $\phi$ admissible. Let $\delta$ be a small, positively oriented circle about $\zeta$ in $\Omega \cap S$ not intersecting $\gamma$ or $\gamma^{\prime}$. Since $\left(\tilde{\phi}(z) /(z-\zeta), S-\{\zeta\}, \gamma^{\prime}\right)$ is $e^{\zeta z} \circ \phi(z)$-admissible, 


$$
(T, \phi)(\zeta)=\left\langle f(z), e^{\zeta z} \circ \phi(z)\right\rangle=\frac{1}{2 \pi i} \int_{\gamma^{\prime}} \frac{f(w) \tilde{\phi}(w)}{w-\zeta} d w .
$$

Writing this integral as the sum of integrals over $\delta$ and $\gamma$, the right member is $f(\zeta) \tilde{\phi}(\zeta)-\omega(\zeta)$.

Theorem 2. For $f \# 0$ in $H$, the mapping $\phi \rightarrow f * \phi$ maps $E$ onto $E$. Further, if $\psi \in E$ and $(\tilde{\psi}, S, \gamma)$ is $\psi$-admissible with the only zeros of $f$ on or interior to $\gamma$ in $\tilde{S}$, and if

$$
\psi_{0}(z)=\frac{1}{2 \pi i} \int_{\gamma} \frac{e^{z w^{\breve{\psi}}(w)}}{f(w)} d w
$$

then $f * \psi_{0}=\psi$.

Proof. For each $z$ in $S$ choose $\gamma_{z}$ so that $\left(\psi, S, \gamma_{z}\right)$ is $\psi$-admissible with the only zeros of $f$ on or interior to $\gamma_{z}$ in $\tilde{S}$ and so that $z$ is in the exterior of $\gamma_{z} \cdot$ Then

$$
\eta(z)=\int_{\gamma_{z}} \frac{\tilde{\psi}(w)}{f(w)(z-w)} d w
$$

defines a function which is analytic in $S$.

$\psi_{0}$ as given in the theorem is entire and of exponential type. Its Borel transform for $z$ large, real and exterior to $\gamma$ is $\int_{0}^{\infty} e^{-z t} \psi_{0}(t) d t$. Writing $\psi_{0}$ as in the theorem, changing the order of integration, and performing the integration with respect to $t$ shows that this transform is $\eta$. Since $\eta$ is analytic in $S,(\eta, S) \in$ $\psi_{0}^{*}$.

Choose $\delta$ in the exterior of $\gamma$ so that $(\eta, S, \delta)$ is $\psi_{0}$-admissible. Then

$$
\begin{aligned}
\left(f * \psi_{0}\right)(z) & =\left\langle e^{z w} f(w), \psi_{0}(w)\right\rangle=\frac{1}{2 \pi i} \int_{\delta} e^{z w} f(w) \eta(w) d w \\
& =\frac{1}{2 \pi i} \int_{\delta} e^{z w} f(w) \frac{1}{2 \pi i} \int_{\gamma} \frac{\tilde{\psi}(t)}{f(t)(w-t)} d t d w \\
& =\frac{1}{2 \pi i} \int_{\gamma} \frac{\tilde{\psi}(t)}{f(t)} \frac{1}{2 \pi i} \int_{\delta} \frac{e^{z w} f(w)}{w-t} d t d w \\
& =\frac{1}{2 \pi i} \int_{\gamma} e^{z t} \tilde{\psi}(t) d t=\left\langle e^{z t}, \psi(t)\right\rangle=\psi(z) .
\end{aligned}
$$

We will use the terminology polynomial-exponential for a function of the form $p(z) e^{\zeta z}$ when $p$ is a polynomial; when $p$ is a monomial we will say such a function is a monomial-exponential. The zero function is to be included as a monomial-exponential. 
Definition 3. For $f \# 0$ in $H$ let $\left\{\zeta_{k}\right\}$ be the zeros of $f$ in $\Omega$ and let $m_{k}+1$ be the order of $\zeta_{k}$. Let $Z(f)=\left\{\left(\zeta_{k^{\prime}} p\right) ; 0 \leq p \leq m_{k^{\prime}} p\right.$ integral $\}$. A polynomialexponential $p_{k}(z) \exp \left(\zeta_{k} z\right)$ belongs to $f$ in $\Omega$ if $p_{k}$ is of degree at most $m_{k}$. The zero function belongs to each $f$ in $\Omega$.

Lemma 3. $\zeta \in \Omega$. $\omega(z)=z^{p} e^{\zeta z} . f \# 0$ is in $H$. Then $f * \omega=0$ if and only if $(\zeta, p) \in Z(f)$.

Proof. Since $\tilde{\omega}(z)=p !(z-\zeta)^{-p-1}, f * \omega=0$ if and only if $\int_{c} e^{z w} f(w)(w-\zeta)^{-p-1} d w=0$ for all $z$ where $c$ is a circle about $\zeta$ in $\Omega$. Hence $f * \omega=0$ if and only if the pth derivative of $e^{z \zeta} f(\zeta)$ with respect to $\zeta$ is zero for all $z$. A simple calculation shows that this condition is equivalent to $f^{(i)}(\zeta)$ $=0$ for $i=0, \ldots, p$, i.e., $\zeta$ is a zero of $/$ of order at least $p+1$.

Theorem 3. $f \# 0$ is in $H$ and $\phi \in E$. Then $f * \phi=0$ if and only if $\phi$ is a finite sum of polynomial-exponentials belonging to $f$ in $\Omega$.

Proof. The fact that such a finite sum satisfies the homogeneous convolution equation is immediate from Lemma 3. Assume now that $f * \phi=0$. Choose $(\tilde{\phi}, S)$ in $\phi^{*}$. Since $f * \phi=0$, the function $\omega$ of Theorem 1 is zero and $\tilde{\phi}=$ $T, \phi$ on $\Omega \cap S . T_{f} \phi / f$ is meromorphic in $\Omega$ since $T_{f} \phi$ and $f$ are analytic there with $f \# 0$. Since $\tilde{\phi}=T_{f} \phi / f$ is analytic in $\Omega \cap S$, the only poles of $T_{f} \phi / f$ in $\Omega$ are in the compact set $\Omega \cap \tilde{S}$. Hence $T_{f} \phi / f$ has only a finite number of poles in $\Omega$. Let $P_{k}$ denote the principal part of $T, \phi / f$ at $\zeta_{k}$. Then if $(\tilde{\phi}, S, \gamma)$ is $\phi$ admissible,

$$
\phi(z)=\frac{1}{2 \pi i} \int_{\gamma} e^{z t \tilde{\phi}}(t) d t=\frac{1}{2 \pi i} \int_{\gamma} e^{z t} \frac{T, \phi(t)}{f(t)} d t=\frac{1}{2 \pi i} \int_{\gamma} e^{z t} \sum P_{k}(t) d t .
$$

It follows that $\phi$ is a finite sum of polynomial-exponentials belonging to $f$ in $\Omega$. Since $\Sigma P_{k}$ vanishes at infinity and is analytic in $S$, it follows that $\tilde{\phi}=\Sigma P_{k}$ and $\tilde{\phi}$ is rational. This observation and our proof give the following corollary.

Corollary. Let $f * \phi=0$ where $f \# 0$ is in $H$ and $\phi \in E$, and let $(\tilde{\phi}, s) \epsilon$ $\phi^{*}$. Then $\tilde{\phi}$ is a rational function and $\tilde{\phi}=T, \phi / f$ in $\Omega$.

It is worth noting here that $T_{f} \phi / f$ is independent of $f$ in the sense that if $f * \phi=g * \phi=0$ for $f$ and $g$ nonzero in $H$, then $T_{f} \phi / f=T_{g} \phi / g$. That this holds is a consequence of the fact that each of $T_{f} \phi / f$ and $T_{g} \phi / g$ is equal to $\tilde{\phi}$.

Corollary. If $f \# 0$ is in $H$, then the set of all $\phi$ in $E$ such that $f * \phi=0$ is the direct sum of the subspaces $\left[z^{p} e^{\zeta z}\right]$ where $(\zeta, p) \in Z(f)$.

Proof. The fact that the set of solutions is the sum of the subspaces follows from the theorem. That the sum is direct follows from the linear independence 
of the monomial-exponentials which may be easily established directly or by using the remark following the next definition.

The general solutions of (1) and (2) will be given more explicitly in the next two theorems. It is also clear from Theorem 3 that if $\phi \neq 0$ satisfies a convolution equation $f * \phi=0$ for some $f \# 0$, then $\phi$ satisfies a similar equation where $f$ is a polynomial of minimal degree; this will be made more explicit in Lemma 4.

Definition 4. For $f \# 0$ in $H$, let $c_{k}$ be a circle in $\Omega$ about zero $\zeta_{k}$ of $f$ in $\Omega$ containing no other zero of $f$ on or inside itself. For each $k$ and each nonnegative integer $b$ define $F_{k b}$ on $E$ by

$$
F_{k b}(\phi)=\frac{1}{2 \pi i} \int_{c_{k}} \frac{\left(t-\zeta_{k}\right)^{b} T, \phi(t)}{f(t)} d t .
$$

It follows easily from Lemma 3 and Theorem 1 that $F_{k b}\left(z^{p} \exp \left(\zeta_{q} z\right)\right)=$ $b ! \delta_{p b} \delta_{q k}$ for $0 \leq p \leq m_{q}$.

Definition 5. If $\phi(z)=\sum_{k=1}^{n} p_{k}(z) \exp \left(\lambda_{k} z\right)$ where the $\lambda_{k}$ are distinct and in $\Omega$ and each $p_{k}$ is a nonzero polynomial of degree $d_{k}$, then $f_{\phi}(z) \equiv \Pi_{k=1}^{n}\left(z-\lambda_{k}\right)^{d_{k}+1}$ and $\sigma(\phi)=Z\left(f_{\phi}\right)$.

Theorem 4. $/ \# 0$ is in $H$ and $\phi \in E$. Then $f * \phi=0$ if and only if

$$
\phi(z)=\sum_{\left(\zeta_{k}, b\right) \in Z(f)} \frac{F_{k b}(\phi)}{b !} z^{b} e^{\zeta_{k} z}
$$

$Z(f)$ may be replaced by $\sigma(\phi)$ when $\phi \neq 0$.

Proof. The result follows immediately from the representation of $\phi$ given in the proof of Theorem 3 and the observation that

$$
P_{k}(z)=\sum_{b=0}^{d_{k}} F_{k b}(\phi)\left(z-\zeta_{k}\right)^{-b-1}
$$

Theorem 5. $f \# 0$ is in $H . \phi \in E$ and $\psi \in E$. Then $f * \phi-\psi$ if and only if

$$
\phi(z)=\psi_{0}(z)+\sum_{\left(\zeta_{k}, b\right) \in Z(f)} \frac{F_{k b}\left(\phi-\psi_{0}\right)}{b !}-z^{b} e^{\zeta_{k} z}
$$

where $\psi_{0}$ is as given in Theorem 2. $Z(f)$ may be replaced by o $\left(\phi-\psi_{0}\right)$ when $\phi \neq \psi_{0}$.

Proof. The result is immediate from Theorems 2 and 4.

It follows from Theorem 1 , that if $f$ and $g$ are in $H$ and $\phi$ is in $E$, then $\langle g, f * \phi\rangle-\langle g f, \phi\rangle$ since $T, \phi$ is in $H$. Hence the multiplication mapping $g$ $\rightarrow g f$ in $H$ is adjoint to the convolution mapping $\phi \rightarrow f * \phi$ in $E$. Since this 
adjoint maps $H$ into itself, the convolution mapping is weakly continuous and so continuous in the Mackey topology on $E$; since $H$ is reflexive it is continuous in the strong topology. When $f \# 0$, the convergence of a sequence $\left\{g_{n} /\right\}$ in $H$ implies the convergence of $\left\{g_{n}\right\}$. As a result, the adjoint has a continuous inverse when $f \# 0$ and the convolution mapping is onto $E$ as we have seen in Theorem 2.

Suppose that $f=\left\{f_{i}\right\}$ with $f_{i}$ in $H_{i}$ and $\phi=\Sigma_{1}^{n} \phi_{i}$ with $\phi_{i}$ in $E_{i} \phi_{i}$ may be represented as $(2 \pi i)^{-1} \int e^{z w} \tilde{\phi}(w) d w$ where the integral is taken over a closed curve in $\Omega_{i}$ enclosing the singularities of $\tilde{\phi}$ in $\Omega_{i}$. From Theorem 1 it follows that $f_{i} * \phi_{i}$ is in $E_{i}$ so $\Sigma_{1}^{n} f_{i} * \phi_{i}$ is the direct sum representation of $f * \phi$. Consequently, the general solution of (1) is the direct sum of the solutions of the equations $f_{i} * \phi_{i}=0$; these are determined by Theorem 3 when $f_{i} \neq 0$ and are all of $E_{i}$ whe $7 f_{i}=0$. If $\omega$ is in $\Omega_{i}$, the set of functions $\left\{z^{p} e^{\omega z}\right\}$ where $p$ runs through the nonnegative integers can be seen to be total in $E_{i}$ by the HahnBanach and Cauchy theorems. It follows that the set of all solutions of (1) is a direct sum of closed subspaces generated by monomial-exponentials.

In general, convolution by $f$ maps $E$ onto the direct sum of the $E_{i}$ for which $f_{i} \neq 0$. If $\psi=\Sigma_{1}^{n} \psi_{i}$ and $\psi_{i}=0$ when $f_{i}=0$, then the general solution of (2) is the direct sum of the solutions of the equations $f_{i} * \phi_{i}=\psi_{i}$. Clearly, solutions of (2) do not exist when $f_{i}=0$ and $\psi_{i} \neq 0$ for some $i$.

4. Harmonic analysis in $E$. In this section we will record intrinsic properties of functions in $E$ that satisfy homogeneous convolution equations. We will also examine the closed translation-invariant subspaces of $E$ and their relation to the closed ideals in $H$.

The principal ideal in $H$ generated by $/$ will be denoted by $(f)$. The set of solutions in $E$ of (1) will be denoted by $K(f) . S^{\perp}$ will, as usual, denote the annihilator or orthogonal space of the subspace $S . r(\phi)$ is the closed span in $E$ of the translates of $\phi$.

Lemma 4. $\phi$ in $E$ is a nonzero finite sum of polynomial-exponentials. Then $f * \phi=0$ if and only if $f / f_{\phi} \in H$.

Proof. Assume that $\phi$ and $f_{\phi}$ have the form given in Definition 5. If $f * \phi$ $=0$, then $\tilde{\phi} \tilde{\phi}=T, \phi$ by Theorem 1 and $T_{f} \phi \in H$. If $\lambda_{k}$ is a pole of $\tilde{\phi}$ of order $d_{k}+1$, then either $f$ is the zero function in the component of $\Omega$ containing $\lambda_{k}$ or $f$ has a zero at $\lambda_{k}$ of finite order at least $d_{k}+1$. It follows that $f / f_{\phi} \in H$. Conversely, if $f / f_{\phi} \in H$, then either $f$ is the zero function in the component containing $\lambda_{k}$ or $\lambda_{k}$ is a zero of $f$ of order at least $d_{k}+1$. Hence $f * \phi=0$ by Lemma 3. 
Theorem 6. If $\phi \in E$, then each of the following conditions implies eacb of the others:

1. $f * \phi=0$ for some $f \# 0$ in $H$.

2. $\phi$ is a finite sum of polynomial-exponentials.

3. $r(\phi)$ is finite dimensional and is spanned by the monomial-exponentials it contains.

Proof. The first condition implies the second by Theorem 3. The second implies the first by choosing $f=1$ when $\phi=0$ and $f=f_{\phi}$ when $\phi \neq 0$.

The third condition obviously implies the second. We now show the second condition implies the third. The implication holds when $\phi=0$ since we have included the zero function as a monomial-exponential. Suppose that $\phi \neq 0$ and $\phi$ has the form given in Definition 5. Let $W$ denote the linear span in $E$ of the monomial-exponentials $z^{p} \exp \left(\lambda_{k} z\right)$ where $\left(\lambda_{k}, p\right) \in \sigma(\phi)=Z\left(f_{\phi}\right)$. It is obvious that $\lambda(\phi) \subset W$ and so $x(\phi)$ is finite dimensional. Since the generators of $W$ are linearly independent, the dimension of $W$ is $N \equiv n+\Sigma_{1}^{n} d_{k}$. To show that $r(\phi)=$ $W$ we will exhibit $N$ linearly independent translates of $\phi$.

Choose $\eta$ in $C$ so that the numbers $\exp \left(\eta \lambda_{i}\right)$ are distinct for $\mathrm{i}=1, \ldots, n$. We assert that the translates $\phi_{k \eta}$ for $k=0, \ldots, N-1$ form a linearly independent set. Suppose otherwise, so that there are $c_{k}$, for $k=0, \ldots, N-1$, not all zero with $\Sigma c_{k} \phi(z+k \eta)=0$. Let $b(z)=\Sigma c_{k} z^{k}$ and $f(z)=b\left(e^{\eta} z\right)$. Our assumption on the $c_{k}$ insures that $f \# 0$ and that $f * \phi=0$. Lemma 4 implies that each $\lambda_{k}$ is a zero of $f$ of order at least $d_{k}+1$. This fact together with the choice of $\eta$ insures, by differentiating $f(z)=b\left(e^{\eta z}\right)$, that $b$ has distinct zeros $\exp \left(\eta \lambda_{i}\right)$, the sum of whose orders is $N$. This is impossible since $b$ is of degree $N-1$. We conclude that $W=r(\phi)$ and the second condition implies the third.

Corollary. If $\Omega$ is connected, then $r(\phi)$ is finite dimensional or $r(\phi)=E$.

Proof. If $\phi$ is a sum of polynomial-exponentials, then $r(\phi)$ is finite dimensional by the theorem. If $\phi$ is not such a sum, $g * \phi=0$ must imply that $g=0$; i.e., $\left\langle g, \phi_{\zeta}\right\rangle=0$ for all $\zeta$ implies $g=0$. In this case, $r(\phi)=E$ by the HahnBanach Theorem.

Corollary. $r(\phi)$ is a finite direct sum of closed subspaces spanned by monomial-exponentials.

Proof. If $\phi=\Sigma_{1}^{n} \phi_{i}$, it is easily verified that $\lambda(\phi)=\bigoplus_{1}^{n} \gamma\left(\phi_{i}\right)$ where $\lambda\left(\phi_{i}\right)$ is the closed span of the translates of $\phi_{i}$ in $E_{i}$. The corollary follows from the preceding corollary and the theorem upon recalling that each $E_{i}$ is the closed span of an infinite sequence of monomial-exponentials.

Corollary. $\lambda(\phi) \neq E$ if and only if $f * \phi=0$ for some $f \neq 0$ in $H$. 
Proof. If $\gamma(\phi) \neq E$, the Hahn-Banach Theorem insures the existence of an $f \neq 0$ with $\left\langle f, \phi_{\zeta}\right\rangle=0$ for all $\zeta$, i.e., $f * \phi=0$.

Suppose that $f \neq 0$ and $f * \phi=0$. Then $f_{i} * \phi_{i}=0$ for some $f_{i} \neq 0$. Then $r\left(\phi_{i}\right)$ is finite dimensional in $E_{i}$, and $r(\phi) \neq E$.

Lemma 5. For $f$ in $H, K(f)=(f)^{\perp}$. For $f$ and $g$ in $H, f / g \in H$ if and only if $K(g) \subset K(f)$.

Proof. The first conclusion follows immediately from a general theorem concerning adjoint mappings. More simply, $\phi \in(f)^{\perp}$ if and only if $\langle g /, \phi\rangle=$ $\langle g, f * \phi\rangle=0$ for each $g$ in $H$; i.e., if and only if $f * \phi=0$.

$f / g \in H$ if and only if $(f) \subset(g)$. It is easily verified that $(f)$ is closed in $H$, so this condition is equivalent to $(g)^{\perp} \subset(f)^{\perp}$; i.e., $K(g) \subset K(f)$.

Theorem 7. If $T$ is a closed translation-invariant subspace of $E$ and $g$ is a greatest common divisor of the functions in $T^{\perp}$, then $T=K(\mathrm{~g})$ and $T$ is the direct sum of closed subspaces spanned by monomial-exponentials.

Proof. It is to be understood that $g$ is to be taken as zero in any component of $\Omega$ in which all the functions in $T^{\perp}$ are zero.

We may write $T=\bigoplus T_{i}$ where each $T_{i}$ is a closed subspace of $E_{i}$. That $T_{i}$ is translation invariant follows from Lemma 1 . Hence it suffices to prove the the orem under the assumption that $\Omega$ is connected. Since $T$ is invariant and closed the following statements are equivalent: $\phi \in T ; \phi_{\zeta} \in T$ for all $\zeta$; $\left\langle T^{\perp}, \phi_{\zeta}\right\rangle=0$ for all $\zeta ; f * \phi=0$ for each $f$ in $T^{\perp}$. It follows that $T=\bigcap \kappa(f)$ where the intersection is over all $f$ in $T^{\perp}$.

If $T^{\perp}$ contains only the zero function, then, choosing $g=0$, we have $T=$ $E=K(g)$. Suppose now that $T^{\perp} \notin\{0\}$. We assert that $T=K(g)$. If $f \in T^{\perp}$, then $K(g) \subset K(f)$ by Lemma 5 since $g$ divides $f$. Hence $K(g) \subset T$. Suppose that $\phi \in T$ so that $f * \phi=0$ for each $/$ in $T^{\perp}$. Since $T^{\perp} \neq\{0\}$, it follows from Theorem 3 that $\phi$ is a sum of polynomial-exponentials. If $\phi=0$, then $\phi \in K(g)$. If $\phi \neq 0$, then $f / f_{\phi} \in H$ for each $/$ in $T^{\perp}$ by Lemma 4; that is, $f_{\phi}$ is a common divisor of the functions in $T^{\perp}$. Therefore $f_{\phi}$ divides $g$, and by Lemma 4 we have $g * \phi=0$ and $\phi \in K(g)$. Hence $T=K(g)$. We have seen earlier that $K(g)$ is a direct sum of closed subspaces spanned by monomial-exponentials.

Theorem 8. There is a one-to-one correspondence between the set of all closed ideals in $H$ and the set of closed translation-invariant subspaces of $E$. Ideals and subspaces correspond to their annibilators.

Proof. Using Theorem 7, we will prove the known fact [8, for example] that the closed ideals in $H$ are the principal ideals. We know that the 
principal ideals are closed. Suppose that $I$ is a closed ideal, and let $T=I^{\perp}$. Then $T$ is closed and $T^{\perp}=l$. We assert that $T$ is invariant. For suppose that $\phi \in T$. Then $\langle f, \phi\rangle=0$ for each $f$ in $I$. Then $0=\left\langle e^{\zeta w} f(w), \phi(w)\right\rangle=\left\langle f, \phi_{\zeta}\right\rangle$ for all $\zeta$ and each $f$ in $I=T^{\perp}$. Since $T$ is closed, $\phi_{\zeta} \in T$, proving the assertion. Since $T$ is closed and invariant, it follows from Theorem 7 that $T=K(g)$ for some $g$ in $H$. Hence $I=T^{\perp}=K(g)^{\perp}=(g)$ by Lemma 5 .

The mapping $(g) \rightarrow K(g)$ into the invariant subspaces of $E$ is surjective by Theorem 7. That the mapping is bijective follows from Lemma 5. For if $K(g)=$ $K(f)$, then $(g)^{\perp}=(f)^{\perp}$ and $(g)=(f)$. Lemma 5 also shows that the correspondence is one of annihilators.

5. Mapping $H$ into $E$ by convolution. For completeness we will briefly examine the mapping of $H$ into $E$ defined by $f \rightarrow f * \phi$ for a fixed $\phi$ in $E$. For simplicity we will assume that $\Omega$ is connected and will denote the mapping by $* \phi$. We have noted that it follows from Theorem 1 that $\langle g, f * \phi\rangle=\langle g f, \phi\rangle$ when $g$ and $f$ are in $H$. It follows that $\langle g, f * \phi\rangle=\langle f, g * \phi\rangle$ and $* \phi$ is selfadjoint.

We assert that if $f \neq 0$ and $f * \phi$ is a finite sum of polynomialexponentials, then $\phi$ is also such a sum. For if $f * \phi$ is such a sum, $g *(f * \phi)=0$ for some $g \neq 0$ in $H$. Then $\left\langle e^{z w} g(w) f(w), \phi(w)\right\rangle=\left\langle e^{z w} g(w),(f * \phi)(w)\right\rangle=0$ and $(g f) * \phi=$ 0 . Since $g f \neq 0, \phi$ is a finite sum of polynomial-exponentials.

Consider first the mapping $* \phi$ when $\phi$ is not a finite sum of polynomialexponentials. It follows from Theorem 3 that if $f * \phi=0$, then $f=0$. Hence $* \phi$ is injective. Since $* \phi$ is selfadjoint, the range of the mapping is weakly dense, and so dense, in $E=r(\phi)$. From the assertion of the preceding paragraph, no nonzero finite sum of polynomial-exponentials is in the range. It follows that the range is not all of $E$ and is not closed.

Consider now the mapping $* \phi$ when $\phi$ is a nonzero finite sum of polynomial exponentials. By Lemma 4 , the kernel of the mapping is $\left(f_{\phi}\right)$. If $R$ denotes the range of the mapping, it follows that $\left(f_{\phi}\right)=R^{\perp}$ since $* \phi$ is selfadjoint. By Lemma 5 and the proof of Theorem $6, R^{\perp \perp}=\left(f_{\phi}\right)^{\perp}=K\left(f_{\phi}\right)=x(\phi)$. Since $x(\phi)$ is finite dimensional and $R \subset R^{\perp \perp}, R$ is finite dimensional and closed. Hence $R=$ $r(\phi)$.

Using Lemma 4 and Theorem 8 , it is easy to verify the equivalence of the following statements where $f \in H$ and $\phi \in E$ : (1) $f * \phi=0$ for some $\phi \neq 0$; (2) $f$ has a zero in $\Omega$; (3) $(n \neq H$.

\section{REFERENCES}

1. R. P. Boas, Entire functions, Academic Press, New York, 1954. MR 16, 914.

2. D. G. Dickson, Analytic mean periodic functions, Trans. Amer. Math. Soc. 110 (1964), 361-374. MR $29 \# 4880$. 
3. D. G. Dickson, Infinite order differential equations, Proc. Amer. Math. Soco 15 (1964), 638-641. MR 31 \#457.

4. J. P. Kahane, Lectures on mean periodic functions, Tata Institute of Fundamental Research, Bombay, 1958.

5. G. Köthe, Dualität in der Funktionentheorie, J. Reine Angew. Math. 191 (1953), 30-49. MR 15, 132.

6. — Topologische lineare Räume. I, Die Grundlehren der math. Wissenschaften, Band 107, Springer-Verlag, Berlin, 1960. MR 24 \#A411.

7. H. Muggli, Differentialgleichungen unendlich hoher Ordnung mit konstanten Koeffizienten, Comment. Math. Helv. 11 ( 1938 ), 151-179.

8. L. A. Rubel and B. A. Taylor, Functional analysis proofs of some theorems in function theory, Amer. Math. Monthly 76 (1969), 483-489. MR 40 \#384.

9. L. Schwartz, Théorie générale des fonctions moyenne-périodiques, Ann. of Math. (2) 48 (1947), 857 $\rightarrow 22$. MR 9, 428.

DEPARTMENT OF MATHEMATICS, UNIVERSITY OF MICHIGAN, ANN ARBOR, MICHIGAN 48104 